\title{
Tendencias de la Mortalidad Materna en el Instituto Materno Infantil, 1950-1980
}

\author{
Dres.: Joanne Klevens B.*, Rodrigo Díaz LI.**
}

\section{INTRODUCCION}

El análisis de un fenómeno a través de series de tiempos permite apreciar su tendencia, descartando las oscilaciones transitorias que se observan en los cortos períodos. Este tipo de estudio, conduce al planteamiento de hipótesis acerca de los factores que posiblemente hayan intervenido directa o indirectamente en las variaciones observadas a través del tiempo.

La mortalidad materna se ha estudiado frecuentemente por su importancia como causa de mortalidad, además de que se utiliza como indicador del nivel de salud. Las estadísticas sobre la mortalidad materna, especialmente del pasado, son muy incompletas. Algunos países reportan la mortalidad materna a partir de 1920 ó

* Instructora Departamento Medicina Preventiva Pontificia Universidad Javeriana.

* Prof. Asistente Departamento Ginecología y Obstetricia Universidad Nacional de Colombia.
1930. El banco de datos de la OMS dispone de información a partir de 1950, aunque sólo para el $30 \%$ de la población mundial (1). Aunque esta información es de difícil interpretación por problemas de registro y clasificación de muertes, especialmente en países con sistemas de información mal desarrollados, se observa una tendencia hacia el descenso.

En los Estados Unidos, los análisis detallados del comportamiento de la mortalidad materna en el país, como a nivel institucional y regional muestran descensos grandes a partir de 1930 (2-5). Este descenso refleja principalmente una reducción de la mortalidad materna directa u obstétrica según la clasificación de la Federación Internacional de Ginecología y Obstetricia.

En Colombia, el estudio de la mortalidad materna ha recibido mucha atención a nivel de diferentes instituciones y con distintos enfoques. 
En el Instituto Materno Infantil de Bogotá, se publicó el primer trabajo sobre el tema en 1954 (6) apareciendo varios trabajos posteriores que en total abarcan los períodos de estudio 1950-1951, 1954-1957 y 1960-1980 (7-12). Algunos trabajos comparan sus datos con los estudios inmediatamente anteriores en cuanto a la magnitud y la distribución de la mortalidad materna por causas detectando oscilaciones que podrían atribuirse a fluctuaciones temporales $y$ no necesariamente a tendencias reales.

Este trabajo tiene por objeto establecer las tendencias de la mortalidad materna en el Instituto Materno Infantil durante el período que abarca tres décadas, 1950-1980.

\section{METODO}

Se revisaron los trabajos publicados sobre mortalidad materna en el Institu to Materno Infantil. $(6-12)$. Se eliminó ur trabajo por no aclarar el período de estudio (13). De cada trabajo se extractó el número total de defunciones maternas, el número de defunciones maternas atribuidas a cada causa, y el número de nacidos vivos y/o número de casos obstétricos por año, cuando este estaba disponible, o para todo el período de estudio. Las causas de muerte materna se agruparon teniendo en cuenta la clasificación general utilizada por la FIGO y las clasificaciones hechas por algunos de los investigadores (10-12). Las entidades identificadas como causa de muerte se agruparon así:

\section{Muerte Materna Directa:}

- Aborto: Se incluyeron ias muertes con cualquier diagnóstico con mención de aborto; sepsis por aborto, hemorragia por aborto, ruptura uterina por aborto y otras complicaciones con mención de aborto.

- Infección: En este grupo se incluyeron muertes con diagnóstico de infección del puerperio (post-parto o postcesárea).

- Toxemia: En este grupo se incluyeron las muertes atribuidas a preclampsia, eclamṕsia, toxicosis gravídica, hemorragia meningea, accidente cerebro vascular, hemorragia hepática e insuficiencia renal aguda. Para efectos de comparabilidad de datos, se analizó también en este grupo muertes atribuidas a EVHC (Enfermedad Vascular Hipertensiva Crónica).

- Hemorragia: En este grupo se incluyeron muertes atribuidas a placenta previa, ruptura uterina (exceptuando aborto) desprendimiento prematuro de placenta, apoplejía uteroplacentaria, hemorragia del alumbramiento, hemorragia por retención de placenta, atonía uterina post-parto, shock obstétri$\mathrm{co}$, embarazo ectópico y muerte por hemorragia sin especificación de causa.

- Otras Causas Directas: Se incluyeron las muertes atribuidas a embarazo molar, tromboembolismo, embolía cerebral, embolía gaseosa, embolía por líquido amniótico, accidente anestésico, accidente quirúrgico, hiperemesis gravídica, shock trasfusional.

\section{Muerte Materna Indirecta:}

Se incluyeron las muertes atribuidas a tuberculosis de cualquier localización, anemia por uncinaria, anemia por paludismo, anemia perniciosa, amebiasis, cardiopatía, insuficiencia cardio-respira- 
toria, accidente cardiovascular, miocarditis, ruptura de aneurisma, infarto mesentérico, tromboflebitis, nefrosis, uremia, nefroesclerosis, afección renal, glomerulonefritis, coma hiperglicémico, feocromocitoma, y tumor maligno de cualquier localización.

\section{Muerte no Obstetrica:}

Se incluyeron las muertes atribuidas a neumonía, bronconeumonía, ileus paraIf́tico, oclusión intestinal, perforación intestinal por ascaris, fiebre tifoidea, colecistitis, hepatitis viral, hepatitis tóxica, úlcera péptica perforada, fractura de base de cráneo, meningitis, encefalitis, tétanos, peritonitis por herida penetrante. por arma cortopunzante e intoxicación por totes o arsenicales.

Las muertes maternas atribuidas a entidades no claramente clasificables en la lista anterior se consideran aparte en el análisis estableciendo las entidades a las que posiblemente se podrían atribuir, así:

- casos atribuidos a infección sin más especificaciones: se analizaron como sugestivos de aborto o de infecciones post-parto.

- casos atribuidos a ruptura uterina sin más especificaciones: se analizaron como sugestivos de aborto o de hemorragia post-parto.

- casos atribuidos a edema pulmonar se analizaron como sugestivos de causas directas. No se intentó clasificarlas en alguno de los subgrupos de caușa directa.

- casos atribuidos a anemia sin más especificaciones: se analizaron como sugestivo de hemorragia post-parto, o de algunas anemias clasificadas en el grupo de causas indirectas.

- caso atribuido a fibrinogenopenia se analizó como sugestivo de hemorragia o infección.

Los casos sin diagnóstico se excluyeron para el análisis de causas, aclarando el número existente en cada período.

En el estudio de Sánchez Torres (11) se excluyeron 5 muertes maternas ocurridas después de los 42 días de puerperio. Los estudios anteriores a este no especifican si se excluyeron o no muertes por esta razón. Para tener en cuenta esta posibilidad, se analizan las causas primero incluyendo estas muertes y luego rexcluyéndolas. Esta consideración se tuvo para los dos Gl timos estudios (11-12).

Para el cálculo de tasas de mortalidad materna en total y por causas se utilizó como denominador el total de nacidos vivos. Para los estudios publicados antes de 1960, este dato se estimó a partir de los casos obstétricos con base en la relación observada entre casos obstétricos y nacidos vivos reportados para los años 1960-1966 utilizando el método de análi. sis de regresión lineal.

Las tasas de mortalidad materna por año reportado se analizaron inicialmente mediante gráfica lineal y luego mediante el método de regresión segmentaria (14). El punto de unión de los dos segmentos utilizados para identificar tendencias se estableció teniendo en cuenta el error de regresión mínimo de los dos segmentos.

La mortalidad por causas se analizó mediante tasas específicas y mediante la mortalidad proporcional. 


\section{RESULTADOS}

Se obtuvo información acerca de la mortalidad materna en el Instituto Materno Infantil a partir de 1950 faltando por reportarse los años $1952-53$ y 195859. La tabla 1, presenta el número de muertes maternas, nacidos vivos y tasa de mortalidad por los años reportados. Estos datos están representados en la gráfica 1.
La gráfica 1 permite distinguir dos períodos de tendencias diferentes; un primer período de rápido descenso de la mortalidad (1950-1957) y luego un período de descenso muy lento con tasas de mortalidad que oscilan alrededor de $29 \times 10.000$. El ajuste de estas tasas mediante el método de regresión permite observar con mayor claridad las dos tendencias (línea punteada).

TABLA No. 1

MUERTES MATERNAS, NACIDOS VIVOS Y TASAS DE MORTALIDAD MATERNA INSTITUTO MATERNO INFANTIL, BOGOTA 1950-1980

\begin{tabular}{|l|c|c|c|}
\hline $\begin{array}{c}\text { Año/periodo } \\
\text { Reportado }\end{array}$ & $\begin{array}{c}\text { No. Defunciones } \\
\text { Maternas }\end{array}$ & $\begin{array}{c}\text { No. Nacidos } \\
\text { Vivos }\end{array}$ & $\begin{array}{c}\text { Tasa de mortalidad } \\
\times \quad 10.000\end{array}$ \\
\hline $1950-1951$ & 100 & $9122^{\star}$ & 109.6 \\
1954 & 52 & $7819^{\star}$ & 66.5 \\
1955 & 48 & $8552^{\star}$ & 56.1 \\
1956 & 44 & $9797^{\star}$ & 44.9 \\
1957 & 30 & $7682^{\star}$ & 39.1 \\
1960 & 53 & 11662 & 45.4 \\
1961 & 37 & 12908 & 28.7 \\
1962 & 29 & 13604 & 21.3 \\
1963 & 37 & 15873 & 16.3 \\
1964 & 36 & 17392 & 20.7 \\
1965 & 44 & 18684 & 24.1 \\
1966 & 77 & 19879 & 38.7 \\
1967 & 72 & 20625 & 34.9 \\
1968 & 71 & 20920 & 33.8 \\
1969 & 63 & 22335 & 26.2 \\
1970 & 64 & 22159 & 28.9 \\
1971 & NI & NI & 26.9 \\
1972 & NI & NI & 24.9 \\
1973 & NI & NI & 38.9 \\
1974 & NI & NI & 28.7 \\
1975 & NI & NI & 34.1 \\
$1971-1975$ & $365(5)$ & 118479 & -- \\
1976 & 59 & 22275 & 26.6 \\
1977 & 60 & 20769 & 29.0 \\
1976 & 53 & 21421 & 24.8 \\
1979 & 40 & 20869 & 19.2 \\
1980 & 51 & 19761 & 26.0 \\
\hline
\end{tabular}

* Estimado a partir de casos obstétricos reportados para esos años.

$\mathrm{NI}=$ No información 
TENDENCIA SECULAR DE LA TASA DE MORTALIDAD MATERNA EN EL

INSTITUTO MATERNO INFANTIL, BOGOTA 1950-1980

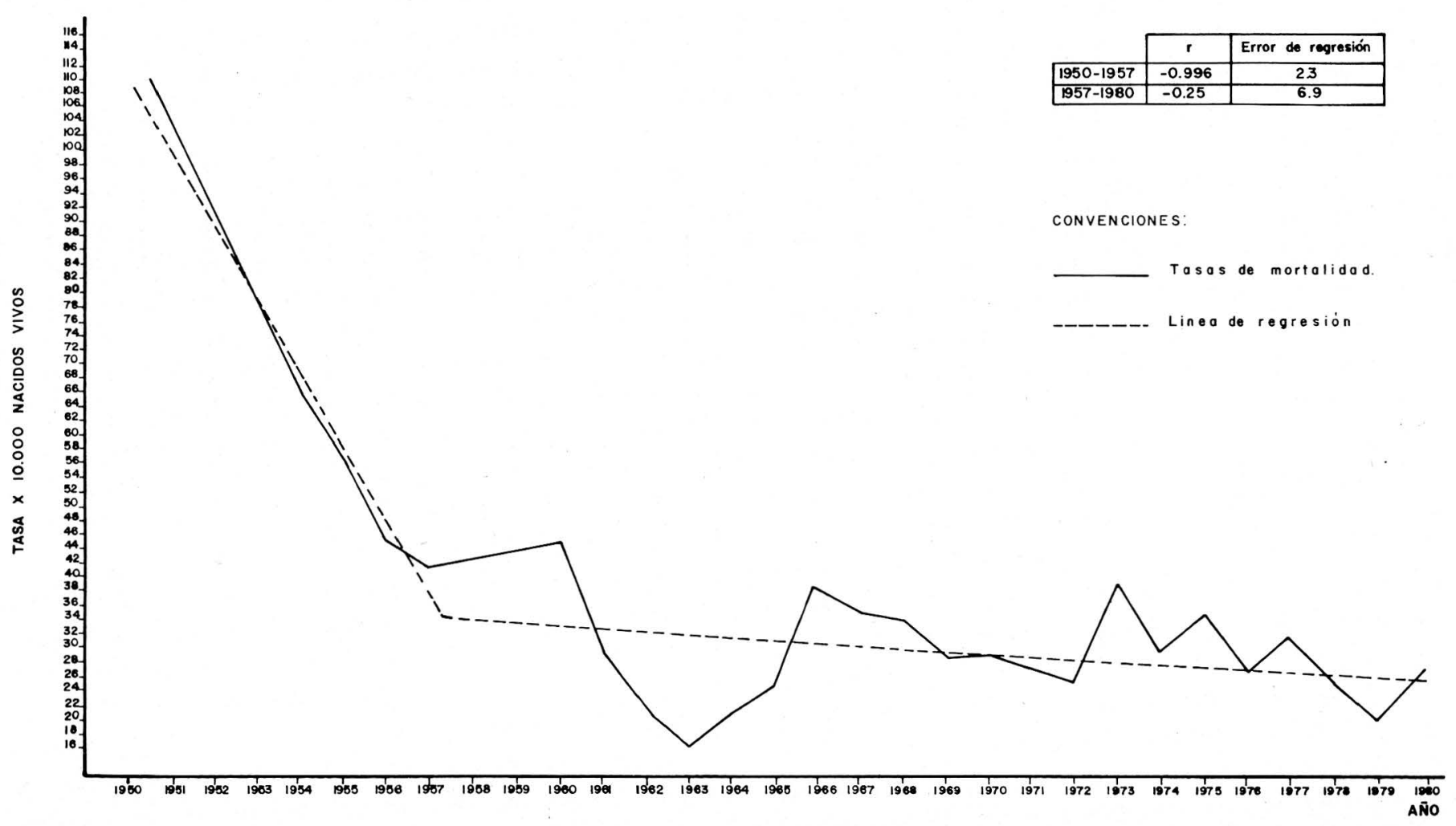


En la tabla 2 y gráfico 2 , se presentan la mortalidad materna agrupada en directa, indirecta y no obstétrica en los diferentes períodos de estudio. Se observa un rápido descenso de los tres grupos de mortalidad, pero especialmente de la mortalidad materna directa, en los perio- dos de 1950 a 1965. El período de 1960-1965 registra la tasa de mortalidad materna directa más baja de los 30 años del estudio. En los siguientes 15 años del estudio la mortalidad materna indirecta permanece estable; la no obstétrica y la directa descienden levemente.

TABLA No. 2

\section{MORTALIDAD MATERNA DIRECTA, INDIRECTA Y NO OBSTETRICA EN DIFERENTES PERIODOS DE ESTUDIO}

\begin{tabular}{|c|c|c|c|c|c|c|c|c|c|}
\hline \multirow{3}{*}{\multicolumn{3}{|c|}{$\begin{array}{c}\text { PERIODO } \\
\text { DE } \\
\text { ESTUDIO }\end{array}$}} & \multicolumn{7}{|c|}{ No. y Tasas $(x$ 10.000) de Mortalidad Materna } \\
\hline & & & \multicolumn{2}{|c|}{ Directa } & \multicolumn{2}{|c|}{ Indirecta } & \multicolumn{2}{|c|}{ No Obstétrica } & \multirow{2}{*}{$\begin{array}{c}\text { No } \\
\text { Clasificable }\end{array}$} \\
\hline & & & No. & Tasa & No. & Tasa & No. & Tasa & \\
\hline 1950 & -1951 & $(6)$ & $72(6)$ & $78.9(82.2)$ & 16 & 17.5 & 8 & 8.8 & 1 \\
\hline 1954 & -1957 & $(7)$ & $133(2)$ & $39.3(39.8)$ & 27 & 8.0 & 6 & 1.8 & 6 \\
\hline 1960 & -1965 & $(8.9)$ & $130(37)$ & $14.4(18.5)$ & $28(2)$ & $3.1(3.3)$ & 25 & 2.8 & 16 \\
\hline 1966 & -1970 & $(10)$ & $306(4)$ & $28.9(29.3)$ & $23(1)$ & $2.2(2.3)$ & 13 & 1.2 & -- \\
\hline 1971 & -1975 & (11) & $315(9)^{\star}$ & $26.6(27.3)$ & 23 & 1.9 & $23(1) \star$ & $1.9(20)$ & 1 \\
\hline 1976 & -1980 & (12) & $208(23)$ & $19.8(22.0)$ & 26 & 2.5 & 6 & 0.6 & -- \\
\hline
\end{tabular}

Nota: Números y tasas de mortalidad entre paréntesis corresponden a causas sin un diagnóstico claro pero sugestivo de alguna causa. Las que aparecen en paréntesis con asterisco corresponden a muertes maternas excluidas por sobrepasar el límite de los 42 días.

Al descomponer la mortalidad materna directa en sus principales causas (tabla 3 y gráfico No. 3), se observa un rápido descenso en las tasas de muerte por hemorragia y toxemia. Las tasas de mortalidad por hemorragia disminuyen rápidamente en el período 1950-1957 para luego estabilizarse. El descenso en las tasas de muerte por toxemia es rápido entre el primero y segundo período del estudio y luego gradual en los últimos 4 períodos. Las tasas de muerte por aborto e infección han permanecido relativamente estables durante los 30 años del estudio, siendo más bajas durante el período 1957-1965.
Para controlar el efecto que produce el descenso general de las tasas de mortalidad materna, se presenta la mortalidad proporcional (tabla y gráfico No. 4). Se observa un aumento progresivo de la proporción de la mortalidad producto de la infección y el aborto, aunque este último desciende un poco en el último período de estudio. Por el contrario, se observa un descenso grande en la proporción de la mortalidad atribuido a hemorragia $y$ en menor magnitud se observa un descenso también en la toxemia. Las causas indirectas también disminuyen en proporción excepto por el último período en que se presenta un aumento. 
GRAFICA No. 2

TASAS DE MORTALIDAD MATERNA DIRECTA, INDIRECTA, Y NO OBSTETRICA EN DIFERENTES PERIODOS DE ESTUDIO, INSTITUTO MATERNO INFANTIL, BOGOTA, 1950-1980

BOGOTA, $1950-1980$

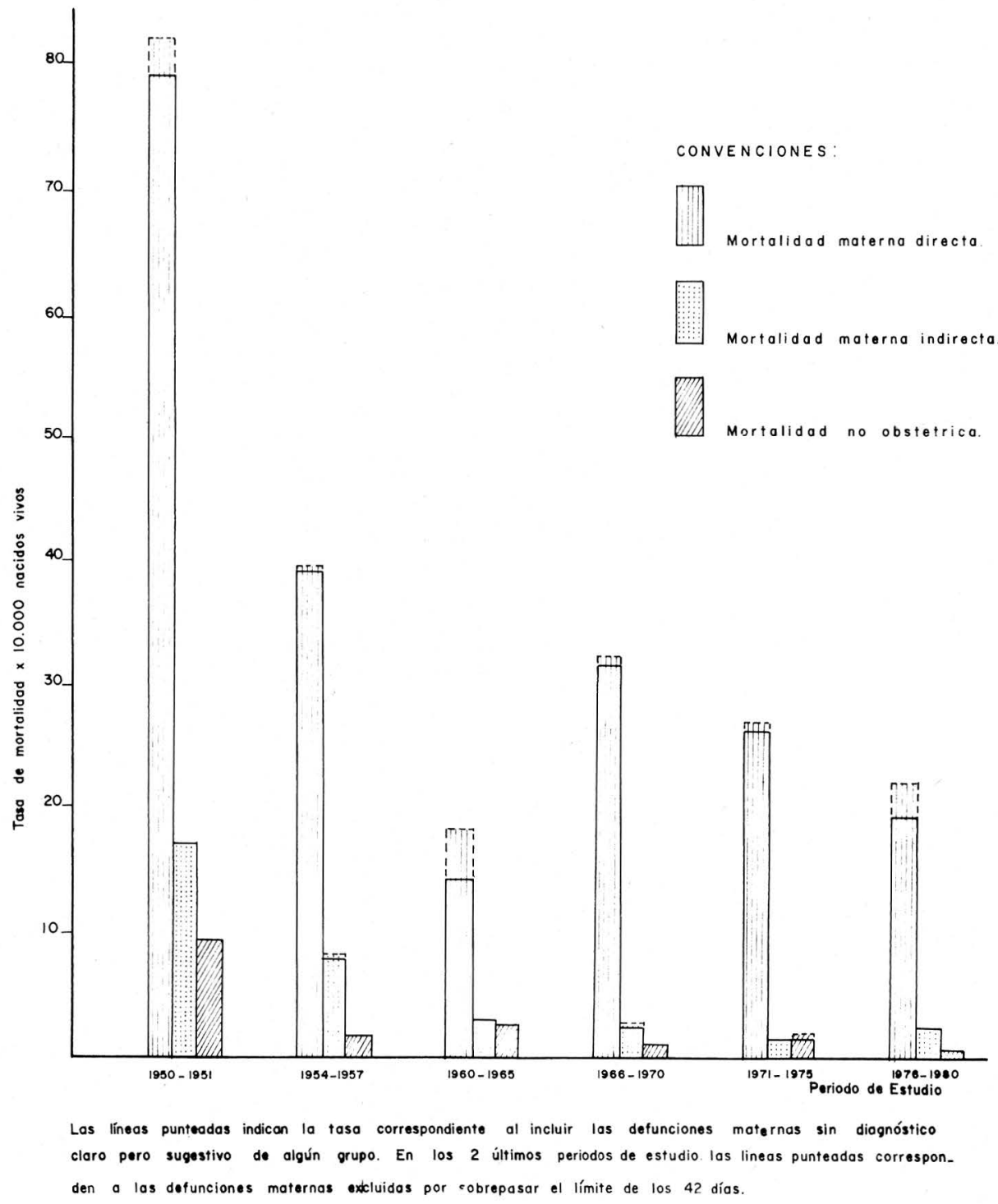




\section{MORTALIDAD MATERNA DIRECTA SEGUN CAUSA EN DIFERENTES PERIODOS DE ESTUDIO INSTITUTO MATERNO INFANTIL. BOGOTA, 1950 - 1980}

\begin{tabular}{|c|c|c|c|c|c|c|c|c|c|c|c|}
\hline \multirow{3}{*}{\multicolumn{2}{|c|}{$\begin{array}{c}\text { PERIODO } \\
\text { DE } \\
\text { ESTUDIO }\end{array}$}} & \multicolumn{10}{|c|}{ No. y Tasas $x 10.000$ de Mortalidad Materna de Causa Directa } \\
\hline & & \multicolumn{2}{|c|}{ Aborto } & \multicolumn{2}{|c|}{ Infección } & \multicolumn{2}{|c|}{ Toxemia } & \multicolumn{2}{|c|}{ Hemorragia } & \multicolumn{2}{|c|}{ Otras Directas } \\
\hline & & No. & Tasa & No. & Tasa & No. & Tasa & No. & Tasa & No. & Tasa \\
\hline $1950-1951$ & $(6)$ & $10(3)$ & $10.9(15.3)$ & $2(3)$ & $2.2(5.5)$ & 25 & 28.5 & 33 & 36.2 & 2 & 2.2 \\
\hline $1954-1957$ & $(7)$ & $17(2)$ & $5.0(5.6)$ & $10(2)$ & $3.0(3.5)$ & 43 & 12.7 & $40(2)$ & $11.8(12.4)$ & 23 & 6.8 \\
\hline $1960-1965$ & $(8-9)$ & $39(28)$ & $4.3(7.4)$ & $3(24)$ & $0.3(3.0)$ & 65 & 7.2 & $14(7)$ & $1.6(2.3)$ & 9 & $1.0^{+}$ \\
\hline $1966-1970$ & $(10)$ & 152 & 14.4 & 49 & 4.6 & 64 & 6.0 & $19(1)$ & $1.8(1.9)$ & $22(3)$ & $2.1(2.4)$ \\
\hline $1971-1975$ & (11) & $163(4) *$ & $13.8(4.1)$ & $57(3)^{\star}$ & $4.8(5.1)$ & 64 & 5.4 & 18 & 1.5 & $13(2)$ & $1.1(1.3)$ \\
\hline $1976-1980$ & (12) & $90(16)$ & $8.5(10.1)^{*}$ & $48(7) *$ & $4.6(5.2)^{*}$ & 44 & 4.2 & 18 & 1.7 & 8 & 0.8 \\
\hline
\end{tabular}

Nota: Números y tasas de mortalidad entre paréntesis corresponden a causas sin un diagnóstico claro pero sugestivo de esa causa. Las que aparecen en paréntesis con asterisco corresponden a muertes maternas excluidas por sobre pasar el límite de los 42 - días.

+ Se excluyeron 7 muertes atribuidas a "edema pulmonar" sin más datos. 
MORTALIDAD MATERNA POR ABORTO, INFECCION POSPARTO, TOXEMIA, HEMORRAGIA Y OTRAS CAUSAS DIRECTAS EN DIFERENTEŚ PERIODOS DE ESTUDIO, INSTITUTO MATERNO INFANTIL, BOGOTA $1950-1980$

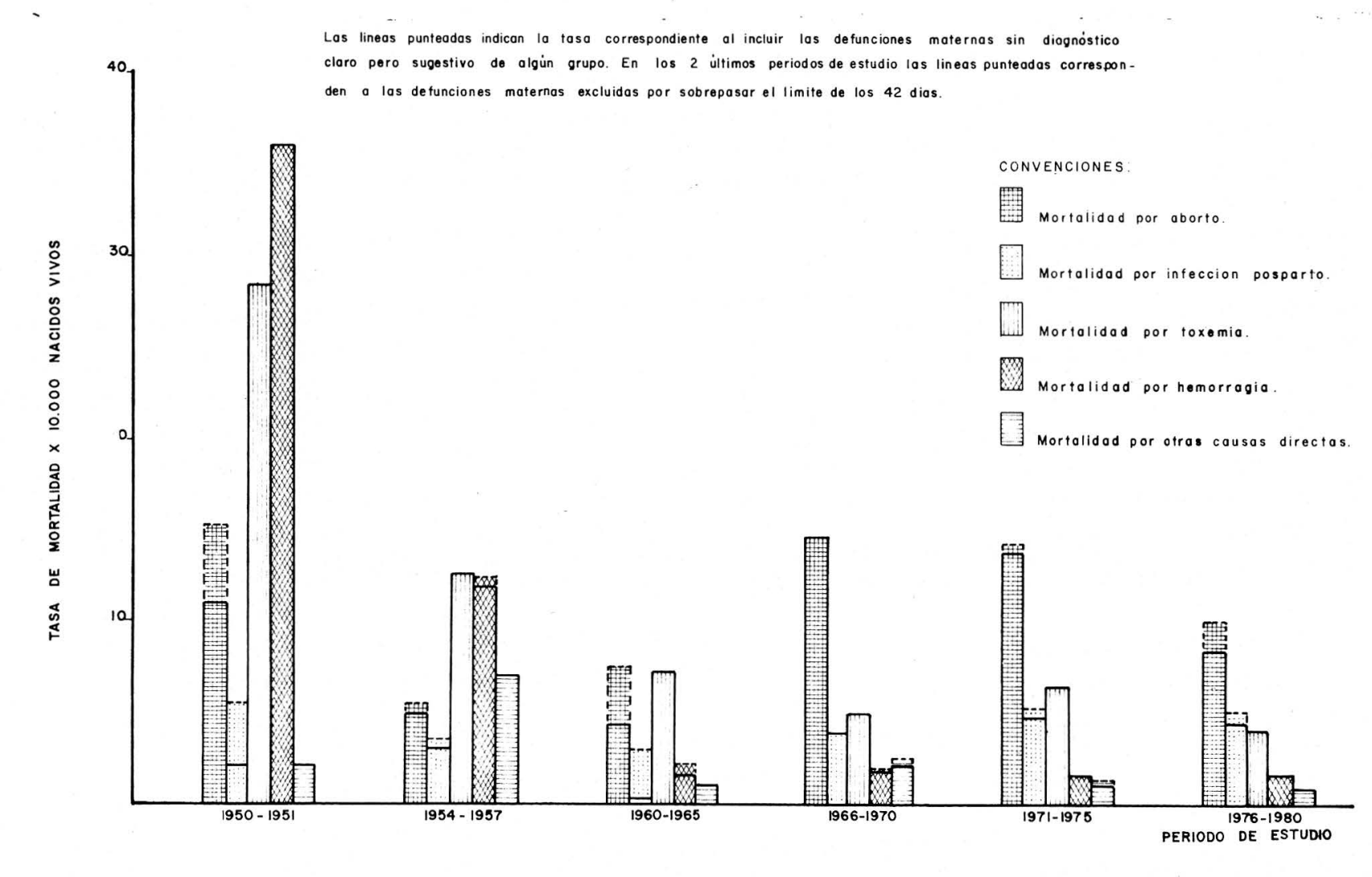

Las lineas punteadas indican la tasa correspondiente al incluir las defunciones maternas sin diognóstico claro pero sugestivo de algun grupo. En los 2 últimos periodos de estudio las lineas punteadas correspon-

den a las defunciones maternas excluidas por sobrepasar el limite de los 42 dios. 
TABLA No. 4

\section{MORTALIDAD MATERNA PROPORCIONAL POR CAUSAS EN DIFERENTES PERIODOS DE ESTUDIO INSTITUTO MATERNO INFANTIL, BOGOTA, 1950 - 1980}

\begin{tabular}{|c|c|c|c|c|c|c|c|c|c|c|c|c|c|c|c|c|c|}
\hline \multirow{3}{*}{\multicolumn{2}{|c|}{$\begin{array}{c}\text { PERIODO } \\
\text { DE } \\
\text { ESTUDIO }\end{array}$}} & \multicolumn{10}{|c|}{$\begin{array}{lllllllll} & i & r & e & c & t & a & s\end{array}$} & \multirow{2}{*}{\multicolumn{2}{|c|}{ Indirectas }} & \multirow{2}{*}{\multicolumn{2}{|c|}{ No Obstétrica }} & \multirow{3}{*}{\begin{tabular}{|c|} 
Mo \\
Casific. \\
No. \\
\end{tabular}} & \multirow{3}{*}{\begin{tabular}{|l} 
Total \\
I:o.
\end{tabular}} \\
\hline & & \multicolumn{2}{|c|}{ Aborto. } & \multicolumn{2}{|c|}{ Infecciosas } & \multicolumn{2}{|c|}{ Toxemia } & \multicolumn{2}{|c|}{ Hemorragia } & \multicolumn{2}{|c|}{ Otras Directas } & & & & & & \\
\hline & & No. & $\%$ & No. & $\%$ & No. & $\%$ & No. & $\%$ & No. & $\%$ & No. & $\%$ & No. & $q$ & & \\
\hline $1950-1951$ & $(6)$ & $10(3)$ & $10.1(13.1)$ & $2(3)$ & $2.0(5.1)$ & 25 & 25.2 & 33 & 33.3 & 2 & 2.0 & 16 & 16.2 & 8 & 8.1 & 1 & 100 \\
\hline $1954-1957$ & $(7)$ & $17(2)$ & $10.1(11.3)$ & $10(2)$ & $6.0(7.1)$ & 43 & 25.6 & 40 & 23.8 & 23 & 13.7 & 27 & 16.1 & 6 & 3.5 & 6 & 174 \\
\hline $1960-1965$ & $(8.9)$ & $39(28)$ & $18.3(31.4)$ & $3(24)$ & $1.4(12.7)$ & 65 & 30.5 & $14(7)$ & $6.6(9.9)$ & 9 & 4.2 & 28 & 13.1 & 25 & 11.7 & $16(7)^{+}$ & 236 \\
\hline $1966-1970$ & $(10)$ & 152 & 43.8 & 49 & 14.1 & 64 & 18.4 & $19(1)$ & $5.5(5.8)$ & $22(3)$ & $6.3(7.2)$ & $23(1)$ & $6.6(6.9)$ & 13 & 3.7 & $\ldots$ & 347 \\
\hline $1971-1975$ & (11) & $163(4) \star$ & $45.3(46.4)$ & $57(3) *$ & $15.8(16.7)$ & 64 & 17.8 & 18 & 5.0 & 13 & 3.6 & 23 & 6.4 & $23(1) \star$ & $6.4(6.7)$ & 1 & 361 \\
\hline$-1976-1980$ & (12) & $90(16) *$ & $34.2(40.3)$ & $48(7) *$ & $18.3(20.9)$ & $44(17)$ & $16.7(23.2)$ & 18 & 6.8 & 8 & 3.0 & $9(17)^{\circ}$ & 3.4 & 6 & 2.3 & $\ldots$ & 263 \\
\hline
\end{tabular}

Nota: Números de defunciones maternas y su proporción correspondiente que aparecen entre paréntesis se refieren a causas sin diagnóstico claro pero sugestivo de algunas causas especificadas. Las que aparecen en paréntesis con asterisco corresponden a muertes maternas excluidas por sobrepasar el límite de los 42 días.

+ En el período 1960 - 1965, se eliminaron del análisis 7 muertes atribuidas a "edema pulmonar"

- Corresponde a muertes atribuidas a EVHC.

Los porcentajes se han caiculado con base en el total de defunciones clasificadas, excluyendo los NO CLASIFICABLES. 
GRAFICA No. 4

MORTALIDAD MATERNA PROPORCIONAL POR CAUSAS EN DIFERENTES PERIODOS DE ESTUDIO, INSTITUTO MATERNO INFANTIL,

\section{BOGOTA 1950 - 1980}

Las lineos punteadas indican la tasa correspondiente al incluir las defunciones maternas sin diagnóstico claro dero sugestivo de algún grupo. En las 2 últimas periodos de estudio las lineas punteadas corresponden a las defunciones maternas excluidas por sobreposar el limite de los 42 dias

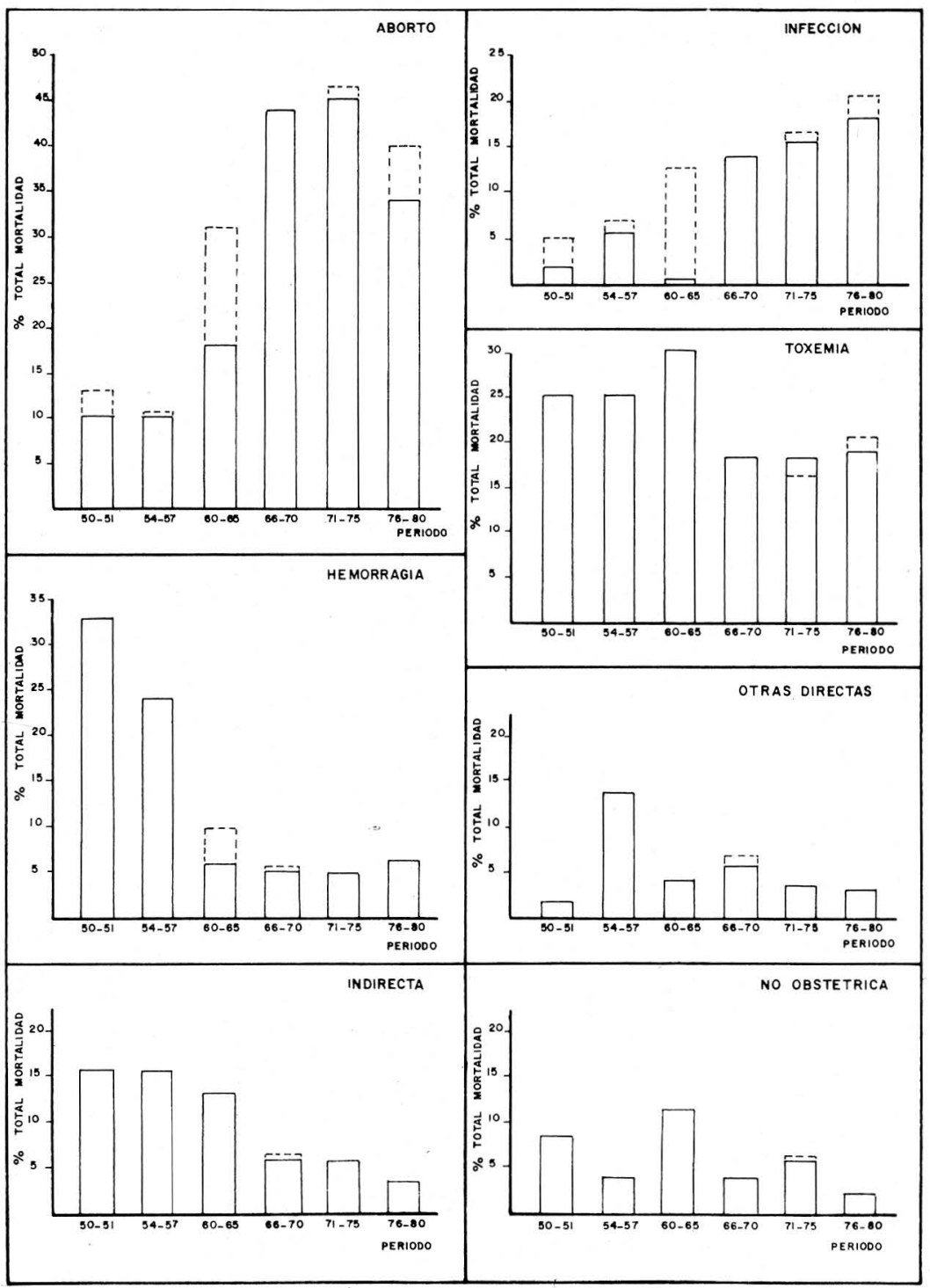




\section{DISCUSION}

Es conveniente considerar algunas características especiales de la información que se presenta para tener más elementos al hacer inferencias.

- Primero: El descenso rápido observado en el período 1951-1960 podría atribuirse a la metodología utilizada para estimar la población de nacidos vivos. Es posible que la diferencia de tendencias entre los dos perfodos no sea tan brusco como se observa.

- Segundo: En un estudio de este tipo, en que se analizan datos recolectados a través de un largo período de tiempo, es necesario considerar posibilidades de error por diferencias en "estilos" diagnósticos y clasificaciones utilizadas en los distintos períodos. Esto se minimizó hasta donde fue posible al estandarizar los criterios de agrupación por causas para la totalidad del período. Además, en aquellas entidades de ciasificación "dudosa", se analizaron los datos con y sin la inclusión de estas muertes.

- Tercero: A través de las tres décadas incluidas en el estudio, es posible que el tipo de población atendida por el Institu to Materno Infantil (I.M.I.) haya sufrido cambios que pudieran explicar las variaciones observadas. Este sería el caso si el I.M.I., hubiera atendido a poblaciones de diferentes estratos en los distintos períodos. Precisamente por esta razón se excluyeron del análisis los estudios posteriores a 1980. Después de este período se inició la transformación del sistema de atención materna en el Distrito para convertir al I.M.I., en centro de atención de alto riesgo. En los períodos anteriores, basándonos en las observaciones hechas por los autores de los estudios de mortalidad materna en el I.M.I., parece que la población conservó características similares. Peralta y colaboradores describen al I.M.I., como "una entidad hospitalaria de caridad" que atendía "cerca del $90 \%$ de la población femenina paupérrima en estado de embarazo" (7). Veinte años más tarde, Múñoz y colaboradores describen la población en similares términos (12), Aunque agregan además la característica de ser sitio de referencia de pacientes de alto riesgo. Estas pacientes también pertenecen a los estratos de bajos recursos que igualmente se atendían, en los períodos anteriores.

Teniendo en cuenta estas observaciones, los datos nos muestran un descenso rápido de la mortalidad materna en el I.M.I., durante el período 1951-1960, y luego un descenso lento después de este período. Al analizar la distribución por causas se observa que la mortalidad materna es, en su mayor proporción, mortalidad por causa directa y esta, a su vez, se distribuye en 4 causas principales: aborto, infección puerperal, toxemia y hemorragia. Se observa además, un descenso importante de la toxemia y la hemorragia, mientras que las tasas de mortalidad por aborto e infección han permanecido relativamente estables. Estos cambios han hecho que se invierta el orden de importancia de estas causas; la hemorragia y la toxemia, principales causas de mortalidad en la década de los 50, han dejado los primeros lugares al aborto y a la infección en la última década del estudio.

La disminución de la mortalidad materna por hemorragia y toxemia es fácilmente explicable. Ha variado el comportamiento reproductivo, especialmente en 
Bogotá, observándose un aplazamiento de la edad del primer embarazo y una disminución de la fecundidad total (15). Aunqưe aún no se ha llegado a una cobertura del $100 \%$ la mayor asistencia al control prenatal y a las instituciones para atención del parto también pueden expliçar esta disminución. A nivel de la institución es importante destacar el mejor control de trabajo de parto que permite tratamientos e intervenciones más oportunas como posibles causas en la disminución de la letalidad por estas causas.

Es llamativa la relativa estabilidad de las tasas de mortalidad por infección posparto, especialmente si se tiene en cuenta el progreso en las técnicas de asepsia, antisepsia y métodos quirúrgicos además, del gran espectro de antibióticos disponibles. Considerando los grandes costos económicos y sociales generados por la infección intrahospitalaria, es recomendable realizar un estudio más profundo que identifique los factores de riesgo de la infección en el I.M.I., como base para establecer las medidas necesarias de prevención y control.

Finalmente, aunque el problema del aborto se ha planteado en repetidas ocasiones y en diferentes ámbitos, siendo la principal causa de mortalidad materna actualmente, debemos resaltar su importancia e insistir en la necesidad de enfrentar el problema y sus soluciones. Los hallazgos de un estudio sobre el problema del aborto a nivel hospitalario en Bogotá (16), muestran que no es la adolescente soltera la que tiene mayor riesgo de recurrir al aborto provocado como método de planificación familiar sino mujeres alrededor de 26.8 años, en su mayoría casadas, con 1 ó más hijos que en el momento de quedar embarazadas no utilizaban métodos anticonceptivos. Los resultados muestran además, que aunque muchas mujeres registraban antecedentes de abortos previos, a su egreso de la institución, menos de la cuarta parte recibía instrucciones acerca de algún método anticonceptivo. Considerando estos hallazgos, las instituciones pueden modificar los riesgos de morbimortalidad por aborto proporcionando información y métodos eficaces de planificación familiar a las embarazadas, a las puérperas y a las mujeres en el período post-aborto. Sin embargo, el descenso radical de la mortalidad por aborto observado en otros países después de su legalización (17) hace pensar en la urgencia de reconsiderar también su legalización en Colombia.

\section{RESUMEN}

El análisis de la mortalidad materna en el Instituto Materno Infantil a través de tres décadas muestra:

- Un descenso rápido de la tasa de mortalidad materna de 1950 a 1960 y un descenso lento de las tasas de 1960 a 1980.

- Que la mayor proporción de la mortalidad en todos los períodos de estudio corresponde a mortalidad materna de causa directa y esta, a su vez, se distribuye en 4 causas principales: aborto, infección puerperal, toxemia $y$ hemorragia.

- Un descenso de las tasas de mortalidad por toxemia y hemorragia, especialmente en el período 1950-1960.

- Relativa estabilidad de las tasas de mortalidad por aborto e infección a 
pesar de los avances en técnicas de asepsia y antisepsia.

- Variación en la importancia relativa de estas 4 causas. En la década de los 50 , la hemorragia y la toxemia ocupaban los primeros lugares. Después de este período, las primeras causas de mortalidad son el aborto y la infección.

\section{BIBLIOGRAFIA}

1. TIETZE, C. Maternal Mortality Excluding Abortion Mortality. World Health Stat. Rep. 30 (4): 312-339. 1977.

2. ROCHAT, RW. Maternal Mortality in the USA. World Health Stat. Q. 34 (1): 2-13, 1981.

3. SACHS, B.P. y Col. Reproductive Mortality in the U.S. JAMA 247 (20): 2789-2792, 1982.

4. JOW-CHING TU, E. Cohort Maternal Mortality, 1917-1972. Amer J. Pub., Health. 69 (10): 1052-1055, 1979.

5. VARNER, M.W. y Col. Maternal Mortality in a major referral hospital. 1962 . 1980. Amer. J. Obstet Gynecol 143 (3): 325-330, 1982.

6. SILVA, CR. Anotaciones sobre Mortalidad Materna en Clínica Obstétrica. Ginec. 5 (5): 41-55, 1954.

7. PERAlta, R., Silva, CR. PEREZ E., RAMIREZ, J. Mortalidad Materna Rev. Col. Obst. Ginec. 9 (1): 1-39, 1958.

8. LOPEZ, R. Morbilidad y Mortalidad en el IMI. Bogotá, 1960-1964. Rev. Col. Ginec. 16 (4): 313-349, 1965.

9. LOPEZ, R. Morbilidad y Mortalidad en el IMI. 1965-66 Rev. Col. Obst. Ginec., 16 (4): 269-282, 1968.
Ante estos resultados, se plantea la posibilidad de reducir aún más la mortalidad materna en el Instituto mediante el estudio de los factores de riesgo de la infección puerperal, el análisis de las medidas pertinentes para su prevención y control y la aplicación de estas medidas en conjunto con las ya conocidas para la prevención y control de morbimortalidad por aborto.

10. ROJAS L., RAMIREZ R., CANTILLO,. J. Mortalidad Materna en el IMI de Bogotá. 1966-1970. Rev. Col.Obst. Ginec. 25 (2): 127-149, 1974.

11. SANCHEZ, F. Mortalidad Materna. Rev. Col. Obst. Ginec. 28 (6): 217-229, 1977.

12. MUÑOZ, L.A., NAÑEZ H., BECERRA E., KLEVENS J. Mortalidad Materna. Instituto Materno Infantil. 1976-1980. Rev. Col. Obst. Ginec. 34 (4): 227 243, 1985.

13. MERA, F. Dos años de Jefatura de Clínica en el Instituto de Protección Materno Infantil. Rev. Col. Ginec. 6 (1): 19-47, 1954.

14. GODFREY K., Simple Linear in Medical Research. N. Engl. J. Med. 313: 16291636, 1985.

15. OCHOA, L.H., RICHARDSON, P., LOAIZA E. Segunda Encuesta Nacional de Prevalencia del Uso de Anticoncepción en Colombia, 1980. Resultados Generales. Corporación Centro Regional de Población, MinSalud. Bogotá, 1981.

16. MEDINA, J., LOPEZ, E.G. A borto Hospitalario en Bogotá. Rev. Col. Obst. Ginec. 31 (1): 2-13, 1980.

17. CATES, W. Legal Abortion: The Public Health Record. Science 215 (26): 128690, 1982. 